\title{
Different prognostic values of individual hematologic parameters in papillary thyroid cancer due to age-related changes in immunity
}

\author{
Kwan Ho Lee*, Eun Young Seok*, Eun Young Kim, Ji Sup Yun, Yong Lai Park, Chan Heun Park \\ Department of Surgery, Kangbuk Samsung Hospital, Sungkyunkwan University School of Medicine, Seoul, Korea
}

\begin{abstract}
Purpose: Several studies have reported the plausible association of neutrophil-to-lymphocyte ratio (NLR) with the prognosis of papillary thyroid cancer (PTC), but the results are inconsistent. In PTC, chronic inflammation is closely related to tumor progression, and the age of the patient has a great influence on prognosis. Therefore, considering the changes in the immune system with aging, we classified the patients according to age and assessed the prognostic value of individual hematologic parameters.

Methods: This retrospective analysis included 1,921 patients who underwent thyroidectomy for PTC. Patients were divided into 2 groups based on their age: Y-group lage $<45$ l and 0 -group lage $\geq 45$ ). Blood counts were measured within 14 days before surgery.

Results: The Y-group consisted of 914 patients aged $<45$ years and the 0 -group consisted of 932 patients aged $\geq 45$. In both groups, the common prognostic factors related to disease recurrence were only 6 or more metastatic lymph node and grossly extrathyroidal extension, and hematologic parameters were different between the 2 groups. High Plateletlymphocyte ratio (PLR) in the Y-group and high NLR in the 0 -group were identified as independent predictors of disease recurrence (NLR: hazard ratio [HR], 3.28; 95\% confidence interval [CI], 1.23-8.73; P = 0.018; PLR: HR, 3.08; 95\% Cl, $1.26-$ 7.52; $P=0.014)$.

Conclusion: The results suggest that changes in immunity with aging may affect prognosis in patients with PTC, and thus hematologic parameters might be employed as prognostic markers depending on the age of the patients.

[Ann Surg Treat Res 2019;96(2):70-77]
\end{abstract}

Key Words: Thyroid neoplasms, Neutrophils, Lymphocytes, Hematologic tests

\section{INTRODUCTION}

Progression to cancer is associated with an impaired function of the immune system [1]. In addition, inflammation plays an important role in cancer development, progression, and metastasis, and is related to tumor prognosis and therapeutic response by mediating interaction with the immune response [2,3]. Peripheral blood-based parameters have been studied as user-friendly and less expensive factors reflecting changes in immune response, and it has been suggested that circulating white blood cells, and changes in the proportions of neutrophils, lymphocytes, and monocytes, may be associated with systemic inflammatory responses [4]. In previous studies, the association of host's immune/inflammatory status with the prognosis of cancer has been reported using hematologic parameters such as absolute monocyte count, absolute lymphocyte count, the neutrophil-to-lymphocyte ratio (NLR), the lymphocyte-to-monocyte ratio (LMR), and the platelet-to-
Received May 30, 2018, Revised July 13, 2018, Accepted August 3, 2018

Corresponding Author: Ji Sup Yun

Department of Surgery, Kangbuk Samsung Hospital, Sungkyunkwan University School of Medicine, 29 Saemunan-ro, Jongno-gu, Seoul 03181, Korea

Tel: +82-2-2001-1722, Fax: +82-2-2001-1883

E-mail: subi0117@naver.com

ORCID code: https://orcid.org/0000-0001-9182-2939
*Kwan Ho Lee and Eun Young Seok contributed equally to this work as first author.

Copyright (c) 2019, the Korean Surgical Society

(c) Annals of Surgical Treatment and Research is an Open Access Journal. All articles are distributed under the terms of the Creative Commons Attribution NonCommercial License (http://creativecommons.org/licenses/by-nc/4.0/) which permits unrestricted non-commercial use, distribution, and reproduction in any medium, provided the original work is properly cited. 
lymphocyte ratio (PLR) [1,5-11].

Papillary thyroid cancer (PTC) is one of the most common cancers and its close relation with chronic inflammation demonstrates a high incidence of thyroid cancer in patients with thyroiditis [5]. Henceforth, numerous studies have investigated the association of PTC with NLR, which is commonly used as an inflammatory marker, and demonstrated the prognostic value of NLR [7-11]. Based on the lack of consistency in the correlation between clinicopathological factors and NLR, and presence of evidence on direct long-term outcomes in patients, the use of NLR as a prognostic marker still remains controversial [12]. Until date, there exist no studies on the prognostic value of LMR as a marker, whereas two studies have reported the prognostic value of PLR as a marker, but the relationship with prognosis remains as unclear $[13,14]$.

Differentiated thyroid cancer is only cancer in which age is considered to be an independent prognostic factor in 7th edition of the American Joint Committee on Cancer (AJCC)/ TNM staging systems [15]. Patients over 45 years of age have a clearly differentiated poor prognosis compared to patients below 45 years of age. Changes in the immune system with an increase in age are thought to be one of the causes of these outcomes [16]. However, there is no research-based evidence to prove this theory.

Therefore, in this study, it was considered that other hematologic parameters should be studied because of the uncertainty of the clinical usefulness of NLR for PTC. In addition, it is assumed that changes in immunity due to aging may have resulted in inconsistent conclusions between the studies on the clinical usefulness of NLR. We sought to clarify the differences between hematologic markers and the clinicopathological factors of PTC patients by classifying them based on patient's age.

\section{METHODS}

\section{Study population}

We performed a retrospective analysis of 1,921 patients, aged $\geq 18$ years who underwent thyroidectomy for PTC by a single surgeon in our hospital between January 2008 and January 2012. Excluding criteria were patients with hematologic disorders, a cancer treatment within past 12 months before surgery, acute myocardial infarction or coronary revascularization within 6 months before surgery and active infection or glucocorticoid usage within 3 months before surgery. Apparently, 1,846 patients (96.1\%) were considered as eligible for further analysis. Patient's clinical characteristics and demographic data were collected through a chart review. We used the Seeplex BRAF ACE detection kit (Seegene, Seoul, Korea) to detect the BRAF V600E mutation in tumor tissues after thyroidectomy. The study protocol was approved by the
Institutional Review Board of Kangbuk Samsung Hospital, the Sungkyunkwan University of Korea, on July 6, 2017 (KBSMC 2017-06-033). The institutional review board waived the need for written informed consent from the participants as the project was deemed to be in the low or negligible risk category in accordance with the Korean National Bioethics Committee 'National Statement on Ethical Conduct in Human Research', 2017.

\section{Blood sample analysis}

Blood samples from all patients were obtained within 14 days before elective thyroidectomy. Blood sampling was performed in the morning with a fasting for more than 8 hours as biochemical tests included in the department's protocol for routine preoperative evaluation were also carried out. It was possible to standardize the known impact of circulating hormones (circadian rhythm) on the number and subtype distribution of the various white blood cell indices. The analysis of thyroid-stimulating hormone (TSH), free thyroxine (free T4), triiodothyronine (T3), and thyroglobulin antigen/antibody $(\mathrm{Ag} / \mathrm{Ab})$ was also carried out. Hematological parameters (i.e., the number of lymphocytes, neutrophils, monocytes, and platelets) were counted by an automated hematology analyzer (XN-5000, Sysmex, Kobe, Japan). LMR was calculated as the ratio of absolute counts between lymphocytes and monocytes, NLR as the ratio of absolute counts between neutrophils and lymphocytes, and PLR as the ratio of absolute counts between platelets and lymphocytes.

\section{Statistical methods}

The endpoints assessed were disease-free survival (DFS), which was calculated as the time between diagnosis and disease relapse, or death from PTC. Receiver operating characteristic (ROC) curve and area under the curve (AUC) analysis were performed to determine the most appropriate cutoff points for the LMR, NLR, and PLR. The score with the maximum sensitivity and specificity was selected as the best cutoff value, which decided the maximum value (sensitivity + specificity - 1) of the Youden index [17].

All statistical analyses were performed using R version 3.3.2 [18-20]. The Chi-square or Fisher exact tests were used for comparing clinicopathologic features between different age groups. DFS rates after surgical treatment were determined using the Kaplan-Meier method. The Cox proportional hazards model was used for univariate and multivariate analyses. All tests were two-sided and $\mathrm{P}<0.05$ was considered statistically significant.

\section{Management of PTC}

Patients under the age of 45 years underwent hemithyroidectomy if there was no clinical suspicion of cervical 
lymph nodes (LNs) metastasis, large tumor size $(>1 \mathrm{~cm})$ or extrathyroidal extension (ETE), and a total thyroidectomy was considered in other cases. The patients underwent singleincision, gasless, and endoscopic transaxillary thyroidectomy depending on the patient's preference. This procedure was not applicable in cases with tumor measuring $4 \mathrm{~cm}$ or more in size, gross maximal ETE, multiple conglomerated LN metastases with extensive invasion of surrounding structures in the

Table 1. Comparisons of the prevalence of clinico-pathological factors between age groups

\begin{tabular}{|c|c|c|c|}
\hline Variable & $\begin{array}{l}\text { Y-group (age < 45) } \\
\quad(n=914)\end{array}$ & $\begin{array}{l}\text { O-group (age } \geq 45) \\
\quad(n=932)\end{array}$ & P-value \\
\hline Sex & & & 0.001 \\
\hline Female & $696(76.1)$ & $769(82.5)$ & \\
\hline Male & $218(23.9)$ & $163(17.5)$ & \\
\hline WBC $\left(10^{9}\right.$ cells/L) & $6.3 \pm 1.7$ & $6.1 \pm 1.7$ & 0.010 \\
\hline Platelets $\left(10^{9}\right.$ cells $\left./ \mathrm{L}\right)$ & $280.1 \pm 62.6$ & $275.8 \pm 68.2$ & 0.154 \\
\hline Neutrophil count $\left(10^{9}\right.$ cells/L) & $3.71 \pm 1.36$ & $3.40 \pm 1.29$ & $<0.001$ \\
\hline Lymphocyte count $\left(10^{9}\right.$ cells/L) & $2.06 \pm 0.56$ & $2.18 \pm 0.64$ & $<0.001$ \\
\hline Monocyte count $\left(10^{9}\right.$ cells/L) & $0.38 \pm 0.13$ & $0.37 \pm 0.14$ & 0.080 \\
\hline LMR & $5.9 \pm 2.2$ & $6.5 \pm 2.5$ & $<0.001$ \\
\hline PLR & $146.7 \pm 48.5$ & $128.1 \pm 45.7$ & 0.019 \\
\hline NLR & $1.9 \pm 0.9$ & $1.7 \pm 0.9$ & $<0.001$ \\
\hline Serum TSH (mIU/L) & $2.0 \pm 2.4$ & $2.0 \pm 1.5$ & 0.910 \\
\hline Free T4 & $1.4 \pm 0.2$ & $1.4 \pm 0.2$ & $<0.001$ \\
\hline T3 & $1.4 \pm 0.2$ & $1.4 \pm 0.2$ & 0.676 \\
\hline Thyroglobulin (ng/mL) & $28.2 \pm 83.7$ & $27.6 \pm 73.4$ & 0.872 \\
\hline Antithyroglobulin $\mathrm{Ab}(\mathrm{ng} / \mathrm{mL})$ & $52.4 \pm 168.8$ & $58.4 \pm 214.6$ & 0.521 \\
\hline Extent of operation & & & $<0.001$ \\
\hline Lobectomy & $269(29.4)$ & $101(10.8)$ & \\
\hline Total thyroidectomy & $645(70.6)$ & $831(89.2)$ & \\
\hline Method of operation & & & $<0.001$ \\
\hline Endoscopic & $634(69.4)$ & $857(92.0)$ & \\
\hline Conventional & $280(30.6)$ & $75(8.0)$ & \\
\hline Tumor size $(\mathrm{cm})$ & $1.0 \pm 0.8$ & $1.0 \pm 0.7$ & 0.187 \\
\hline Multifocality & $194(21.2)$ & $223(23.9)$ & 0.183 \\
\hline Bilaterality & $131(14.3)$ & $174(18.7)$ & 0.014 \\
\hline No. of metastatic LNs excised & $2.2 \pm 4.3$ & $1.3 \pm 3.1$ & $<0.001$ \\
\hline No. of $\mathrm{LNs}$ retrieved & $9.6 \pm 12.3$ & $8.7 \pm 11.1$ & 0.087 \\
\hline $\mathrm{pN}$ status & & & $<0.001$ \\
\hline No & $486(53.2)$ & $631(67.7)$ & \\
\hline $\mathrm{N} 1 \mathrm{a}$ & $294(32.2)$ & $212(22.7)$ & \\
\hline $\mathrm{N} 1 \mathrm{~b}$ & $134(14.7)$ & $89(9.5)$ & \\
\hline No. of metastatic LNs & & & $<0.001$ \\
\hline$\leq 5$ & $794(86.9)$ & $871(93.5)$ & \\
\hline$>5$ & $120(13.1)$ & $61(6.5)$ & \\
\hline Extrathyroidal extension & & & 0.312 \\
\hline No & $472(51.6)$ & $468(50.2)$ & \\
\hline Microscopic & $338(37.0)$ & $336(36.1)$ & \\
\hline Grossly & $104(11.4)$ & $128(13.7)$ & \\
\hline Lymphatic invasion & $37(4.0)$ & $22(2.4)$ & 0.054 \\
\hline Vessel invasion & $18(2.0)$ & $7(0.8)$ & 0.039 \\
\hline BRAF V600E mutation & & & 0.165 \\
\hline Not mutated & 86/401 (21.4) & $63 / 366(17.2)$ & \\
\hline Mutated & $315 / 401(78.6)$ & $303 / 366(82.8)$ & \\
\hline Radioiodine ablation & $457(50.0)$ & $679(72.9)$ & $<0.001$ \\
\hline
\end{tabular}

Values are presented as number $(\%)$ or mean \pm standard deviation.

LMR, lymphocyte-to-monocyte ratio; NLR, neutrophil-to-lymphocyte ratio; PLR, platelet-to-lymphocyte ratio; TSH, thyroid-stimulating hormone; T3, triiodothyronine; free T4, free thyroxine; Ab, antibody; LN, lymph node. 
central compartment, and lateral cervical LN metastasis. The endoscopic transaxillary technique was performed using a single incision and as per the method described in previous literature [21,22]. Routine ipsilateral prophylactic central LN dissection was performed for all patients regardless of tumor size or extent and clinical LN status. If lateral LNM was diagnosed in the preoperative examinations, therapeutic lateral cervical neck (level IIa, III, IV, and Vb, excluding sternocleidomastoid, jugular vein, and accessory nerve) dissection (LCND) was performed. As recommended by the American Thyroid Association (ATA) guidelines [23], prophylactic LCND was not performed.

Postoperative radioactive iodine (RAI) ablation at a dose of 30-150 mCi was performed 4-6 weeks after the operation. RAI was performed when patient's TSH level was above 30 $\mathrm{mIU} / \mathrm{L}$ after either thyroxine withdrawal or recombinant TSH injections. In our institute, RAI ablation was performed in all patients who underwent total thyroidectomy with gross ETE, tumor size larger than $4 \mathrm{~cm}$, cervical LN metastasis, or distant metastases. RAI ablation was also recommended for selected patients with minimal ETE, tumor size $1-4 \mathrm{~cm}$, multiplicity, bilaterality and/or higher risk histologic features (tall cell, hobnail variant, columnar cell carcinoma) according to ATA guidelines [23]. After thyroidectomy, all patients underwent thyroglobulin $\mathrm{Ag} / \mathrm{Ab}$ and TSH, free T4, and T3 measurements, neck ultrasound, whole-body iodine scanning, and chest radiography at 6-month intervals.

\section{RESULTS}

A total of 1,846 PTC patients were included in this analysis. Table 1 shows the comparisons of the clinicopathological characteristics and hematological data of patients in different age groups. The Y-group consisted of 914 patients aged $<45$ years and the O-group consisted of 932 patients aged $\geq 45$. LMR was significantly lower and NLR was higher in the Y-group than the O-group. Hemithyroidectomy and endoscopic surgery were performed more frequently in the Y-group compared to O-group. Instances of cervical LN metastasis, vessel invasion, and tumor bilaterality, which are associated with a poor prognosis, were more common in the O-group.

\section{Cutoff values for the LMR, NLR, and PLR in patients with PTC}

Based on the significant differences in the hematologic markers between the 2 groups, cut-off values were obtained for each group. ROC curve analysis was used to determine the optimal cutoff value for DFS. In the Y-group, the LMR cutoff point was 7.18 (AUC $=0.529$ ), NLR was 2.1 (AUC $=0.562$ ), and PLR was 164.24 ( $A U C=0.560$ ). In the O-group, the LMR cutoff point was 10.42 (AUC $=0.500)$, NLR was 1.99 (AUC $=0.579$ ),

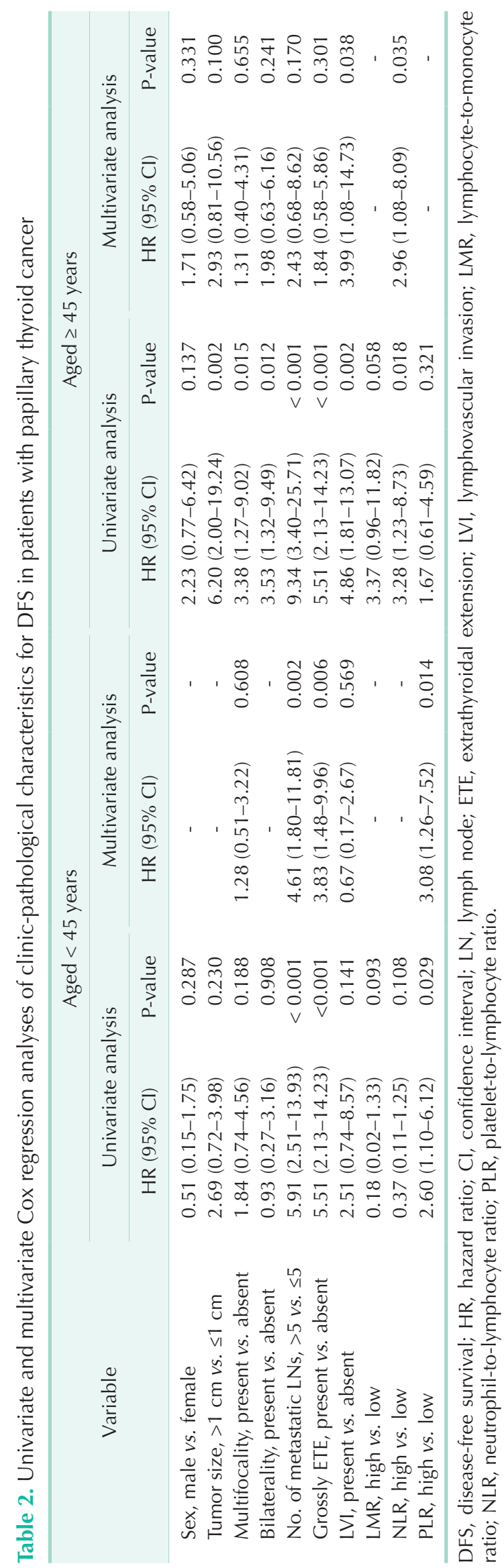


and PLR was 127.41 (AUC $=0.501$ ). Based on these cutoff values, we divided the patients into two groups (low-value vs. highvalue) for survival analysis.

\section{Univariate and multivariate analyses of clinico- pathologic characteristics for DFS in PTC}

The Table 2 shows the Cox regression analysis for DFS. In the univariate analysis, 6 or more metastatic LN (hazard ratio [HR], 5.91; 95\% confidence interval [CI], 2.51-13.93; P < 0.001), grossly ETE (HR, 5.51; 95\% CI, 2.13-14.23; P < 0.001), and high PLR (HR, 2.60; 95\% CI, 1.10-6.12; P = 0.029), were associated with a worse DFS in the Y-group. In the O-group, tumor size (HR, 6.20; 95\% CI, 2.00-19.24; P = 0.002), multifocality (HR, 3.38; 95\% CI, 1.27-9.02; $\mathrm{P}=0.015$ ), bilaterality (HR, 3.53; 95\% CI, 1.32-9.49; $\mathrm{P}=0.012$ ), 6 or more metastatic LNs (HR, 9.34; 95\% CI, 3.4025.71; $\mathrm{P}<0.001$ ), grossly ETE (HR, 4.86; 95\% CI, 1.81-13.07; P = 0.002), lymphovascular invasion (HR, 11.14; 95\% CI, 3.59-34.59; $\mathrm{P}<0.001$ ), and high NLR (HR, 3.28; 95\% CI, 1.23-8.73; P = 0.018) were associated with a worse DFS. No recurrence was noted in the patients without the BRAF V600E mutation in both the groups.

The multivariate analysis with associations emerging from the univariate analysis $(P<0.2)$ revealed that 6 or more metastatic LNs, grossly ETE and PLR status were independent predictors of DFS in the Y-group (6 or more metastatic LNs - HR, 4.61; 95\% CI, 1.80-11.81; P = 0.002, grossly ETE - HR, 3.83; 95\% CI, 1.48-9.96; $\mathrm{P}=0.006$ ), high PLR (HR, 3.08; 95\% CI, 1.26-7.52; $\mathrm{P}=0.014$ ). In the O-group, lymphovascular invasion (HR, 3.99; 95\% CI, 1.08-14.73; P = 0.038) and high NLR (HR, 2.96; 95\% CI, 1.08-8.09; $\mathrm{P}=0.035$ ) were the independent predictors of worse DFS.

\section{DFS in terms of LMR, PLR, and NLR}

After a median follow-up of 75 months (range, 1-115 months), of 1,846 patients, 37 patients (2.0\%) experienced relapse, and 6 patients $(0.3 \%)$ had died. Kaplan-Meier survival curves analysis showed that the DFS in the Y-group patients with low PLR was significantly longer compared to the patients with high PLR ( $P$
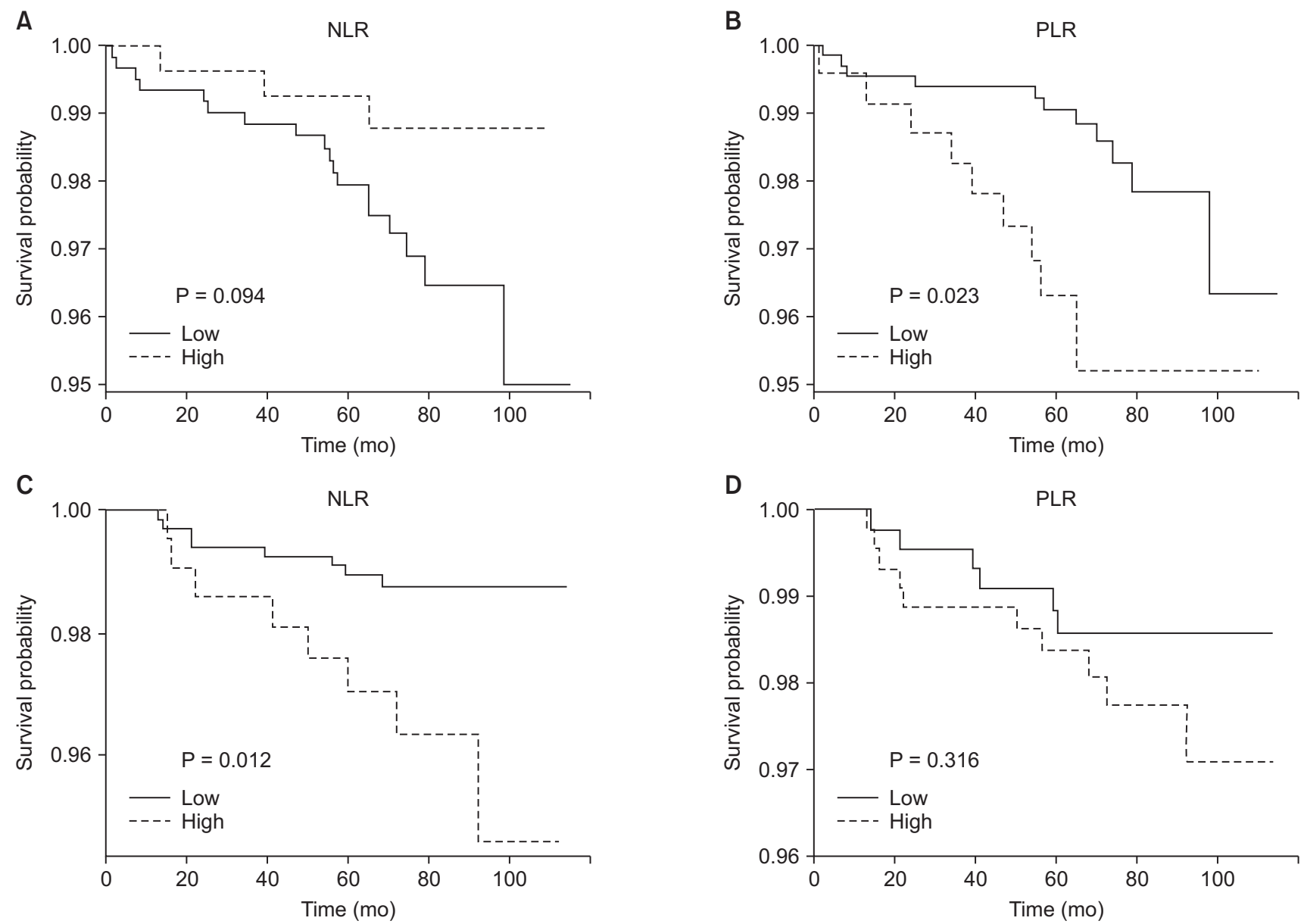

Fig. 1. Hematologic parameters (including LMR, NLR, and PLR) and prognosis. Kaplan-Meier estimates of disease-free survival in the 914 cases of the Y-group (patients aged $<45$ years) (A), the 932 cases of the O-group (patients aged $\geq 45$ years) (B) classified based on the cutoff values of each parameter (high group vs. low group). LMR, lymphocyte-to-monocyte ratio; NLR, neutrophil-to-lymphocyte ratio; PLR, platelet-to-lymphocyte ratio. 
$=0.023)$. On the other hand, in the O-group, the classification according to PLR showed no statistical significance for DFS and low NLR was associated with a good prognosis $(\mathrm{P}=0.012)$ (Fig. 1).

\section{DISCUSSION}

Thyroid cancer has been studied extensively with respect to NLR, and early studies have shown that patients with higher NLR had the higher risk of recurrence and poor prognosis [7$9,11]$. There exist reports on the association of higher NLR with factors suggesting poor prognoses, such as larger tumor size, multifocality, LN metastases, and higher TNM stage [12]. However, other studies have failed to demonstrate the association of NLR with prognosis $[10,11,24]$, and the correlation between clinicopathologic factors and NLR is still inconsistent. Considering the characteristics of thyroid cancer with few recurrences and deaths, a large number of samples are needed to evaluate prognostic factors. Therefore lack of sufficient sample sizes could be one reason of inconsitency. The statistical methodology and heterogeneity of cutoff values between studies can also be causes. However, in our opinion, the most likely cause of inconsistency between the previous reports is the nonconsideration of changes in the immune system based on age and absence of subgroup analysis according to age.

A gradual increase in the proinflammatory status occurs in the process of aging, which was originally called as "Inflammaging" [25]. Though there exists no valid evidence, an imbalance in inflammation homeostasis caused by oxidative stress, proinflammatory cytokines, DNA damage, autophagy, and stem cell aging have been thought to bring about the changes in aging [26]. Inflammation and immunity coexist in the same pathological process and have the same cellular foundation. As age increases, the phenomenon of immunosenescence takes place with decreasing adaptive immunity, while the activation of innate immunity occurs with the rise in proinflammation, called as "Inflamm-aging" [27]. Therefore, Inflamm-aging and immunosenescence should not be considered separately [28]. Some authors have proposed the novel concept of immune/ inflammatory aging (immuno-inflamm-aging), instead of the individual concepts of inflamm-aging and immunosenescence [26].

In this study, the prognostic values of NLR were different between the Y-group and O-group. In the O-group, a significant association of poor prognosis with higher NLR was noted, consistent with previous studies. On the other hand, in the Y-group, higher NLR was tendency for good prognosis, although there was no statistical significance. These conflicting results between the 2 age groups may explain the occurrence of inconsistencies in the prognostic value of NLR in previous studies. It is hypothesized that changes in the immune system with aging may have led to changes in the effect of inflammation on cancer progression or invasiveness. As a result, it could be presumed that the prognostic values of hematologic markers changes with aging.

High PLR in breast cancer has been associated with a poor prognosis [29]. Kim et al. [14] reported that high PLR was significantly associated with lateral LNM in female patients. Ozmen et al. [13] demonstrated the association between PLR and $\mathrm{Tg}$ level and reported that PLR could predict the occurrence of differentiated thyroid cancer. There has been no study on the association of PLR with long-term outcomes. In our study, unlike LMR and NLR, high PLR was associated with a poor prognosis, regardless of age, and demonstrated an independent prognostic value for predicting shorter DFS in patients under 45 years of age (HR, 3.08; 95\% CI, 1.26-7.52; $\mathrm{P}=0.014$ ).

This study has the advantages of the longest median followup period (75 months) and the second largest number of patients $(n=1,846)$ compared to the previous papers on the relationship between NLR and PTC. There are only three literature on direct long-term patient outcomes [9-11], and only the study by Kim et al. [9] demonstrated the prognostic value of NLR. They suggested that the high NLR in the stages III-IV subgroup was a negative prognostic marker for DFS; however the reason behind such an obervation was not provided in their sudies [9]. The results from the reported studies are consistent with our results that NLR has a significant prognostic value in patients over 45 years of age, considering 7 th edition of the AJCC staging system can be categorized as stage III-IV only at the age of 45 years or older and over 45 years of age were included in the present study.

In our study, patients were divided into 2 groups based on the age of 45 years. However, since the age cutoff was changed from 45 to 55 years in 8 th edition of the AJCC/TNM staging systems, it is a limitation of our study to divide the patients into 2 groups based on 45 years old. However, the mortality rate of PTC continues to increase with advancing age beginning at about age 35 years. Therefore, the cutoff of age may not be an important issue in this study, which seeks to compare immunological differences with increasing age.

In conclusion, we demonstrated that PLR in the Y-group and NLR in the O-group are independent predictors of DFS. In addition, this study provides an insight into the appearance of inconsistencies in the results of evaluation of the prognostic value of NLR for PTC in previous studies. It has been suggested that changes in immunity with aging may affect prognosis through progression or metastasis of cancer, and thus the prognostic value of hematologic markers might undergo changes. Therefore, in future studies, it is necessary to focus on changes in the immune response in order to elucidate the cause of aggravation of prognosis according to aging in PTC. Patients with PTC can be expected to receive effective treatment and 
follow-up examination based on the identification of immune markers that can predict the prognosis according to the age of the patient.

\section{CONFLICTS OF INTEREST}

No potential conflict of interest relevant to this article was reported.

\section{REFERENCES}

1. Walsh SR, Cook EJ, Goulder F, Justin TA, Keeling NJ. Neutrophil-lymphocyte ratio as a prognostic factor in colorectal cancer. J Surg Oncol 2005:91:181-4.

2. Iyengar NM, Hudis CA, Dannenberg AJ. Obesity and inflammation: new insights into breast cancer development and progression. Am Soc Clin Oncol Educ Book 2013:46-51.

3. Moore MM, Chua W, Charles KA, Clarke SJ. Inflammation and cancer: causes and consequences. Clin Pharmacol Ther 2010;87:504-8.

4. Coussens LM, Werb Z. Inflammation and cancer. Nature 2002;420:860-7.

5. Seretis C, Gourgiotis S, Gemenetzis G, Seretis F, Lagoudianakis E, Dimitrakopoulos G. The significance of neutrophil/lymphocyte ratio as a possible marker of underlying papillary microcarcinomas in thyroidal goiters: a pilot study. Am J Surg 2013;205:691-6.

6. Proctor MJ, McMillan DC, Morrison DS, Fletcher CD, Horgan PG, Clarke SJ. A derived neutrophil to lymphocyte ratio predicts survival in patients with cancer. Br J Cancer 2012;107:695-9.

7. Liu CL, Lee JJ, Liu TP, Chang YC, Hsu YC, Cheng SP. Blood neutrophil-to-lymphocyte ratio correlates with tumor size in patients with differentiated thyroid cancer. J Surg Oncol 2013;107:493-7.

8. Liu J, Du J, Fan J, Liu K, Zhang B, Wang $S$, et al. The neutrophil-to-lymphocyte ratio correlates with age in patients with papillary thyroid carcinoma. ORL J Otorhinolaryngol Relat Spec 2015;77:109-16.

9. Kim JY, Park T, Jeong SH, Jeong CY, Ju YT, Lee YJ, et al. Prognostic importance of baseline neutrophil to lymphocyte ratio in patients with advanced papillary thyroid carcinomas. Endocrine 2014:46:526-31.

10. Lang BH, Ng CP, Au KB, Wong KP, Wong KK, Wan KY. Does preoperative neutrophil lymphocyte ratio predict risk of recurrence and occult central nodal metastasis in papillary thyroid carcinoma? World J Surg 2014:38:2605-12.

11. Cho JS, Park MH, Ryu YJ, Yoon JH. The neutrophil to lymphocyte ratio can discriminate anaplastic thyroid cancer against poorly or well differentiated cancer. Ann Surg Treat Res 2015;88:187-92.

12. Manatakis DK, Tseleni-Balafouta S, Balalis D, Soulou VN, Korkolis DP, Sakorafas $\mathrm{GH}$, et al. Association of baseline neutrophil-to-lymphocyte ratio with clinicopathological characteristics of papillary thyroid carcinoma. Int J Endocrinol 2017;2017:8471235.

13. Ozmen S, Timur O, Calik I, Altinkaynak K, Simsek E, Gozcu H, et al. Neutrophillymphocyte ratio (NLR) and plateletlymphocyte ratio (PLR) may be superior to $\mathrm{C}$-reactive protein (CRP) for predicting the occurrence of differentiated thyroid cancer. Endocr Regul 2017;51:131-6.

14. Kim SM, Kim EH, Kim BH, Kim JH, Park SB, Nam YJ, et al. Association of the preoperative neutrophil-to-ymphocyte count ratio and platelet-to-lymphocyte count ratio with clinicopathological characteristics in patients with papillary thyroid cancer. Endocrinol Metab (Seoul) 2015:30:494-501.

15. Edge SB, Compton CC. The American Joint Committee on Cancer: the 7th edition of the AJCC cancer staging manual and the future of TNM. Ann Surg Oncol 2010;17:1471-4.

16. Haymart MR. Understanding the relationship between age and thyroid cancer.
Oncologist 2009;14:216-21.

17. Youden WJ. Index for rating diagnostic tests. Cancer 1950;3:32-5.

18. R Core Team. R: A language and environment for statistical computing [Internet]. Vienna: R Foundation for Statistical Computing; c2016 [cited 2018 Feb 20]. Available from: https://www.R-project.org/.

19. Therneau T. A package for survival analysis in S. version 2.38 [Internet]. Vienna: R Foundation for Statistical Computing; c2015 [cited 2018 Feb 20]. Available from: https://CRAN.R-project.org/ package $=$ survival.

20. Therneau TM, Grambsch PM. Modeling survival data: extending the Cox model. New York: Springer; 2000.

21. Linos D. Minimally invasive thyroidectomy: a comprehensive appraisal of existing techniques. Surgery 2011;150:17-24.

22. Kim EY, Lee KH, Park YL, Park CH, Lee CR, Jeong JJ, et al. Single-incision, gasless, endoscopic trans-axillary total thyroidectomy: a feasible and oncologic safe surgery in patients with papillary thyroid carcinoma. J Laparoendosc Adv Surg Tech A 2017;27:1158-64.

23. Haugen BR, Alexander EK, Bible KC, Doherty GM, Mandel SJ, Nikiforov YE, et al. 2015 American Thyroid Association management guidelines for adult patients with thyroid nodules and differentiated thyroid cancer: the American Thyroid Association Guidelines Task Force on thyroid nodules and differentiated thyroid cancer. Thyroid 2016;26:1-133.

24. Yaylaci S, Tosun O, Sahin O, Genc AB, Aydin E, Demiral G, et al. Lack of variation in inflammatory hematological parameters between benign nodular goiter and papillary thyroid cancer. Asian Pac J 
Cancer Prev 2016;17:2321-3.

25. Franceschi C, Bonafe M, Valensin S, Olivieri F, De Luca M, Ottaviani E, et al. Inflamm-aging. An evolutionary perspective on immunosenescence. Ann N Y Acad Sci 2000;908:244-54.

26. Xia S, Zhang X, Zheng S, Khanabdali $\mathrm{R}$, Kalionis $\mathrm{B}, \mathrm{Wu}$ J, et al. An update on inflamm-aging: mechanisms, prevention, and treatment. J Immunol Res 2016;2016:
8426874.

27. Salminen A, Huuskonen J, Ojala J, Kauppinen A, Kaarniranta K, Suuronen T. Activation of innate immunity system during aging: NF-kB signaling is the molecular culprit of inflamm-aging. Ageing Res Rev 2008;7:83-105.

28. Pes GM, Lio D, Carru C, Deiana L, Baggio G, Franceschi C, et al. Association between longevity and cytokine gene poly- morphisms. A study in Sardinian centenarians. Aging Clin Exp Res 2004;16: 244-8.

29. Krenn-Pilko S, Langsenlehner U, Thurner EM, Stojakovic T, Pichler M, Gerger A, et al. The elevated preoperative platelet-tolymphocyte ratio predicts poor prognosis in breast cancer patients. Br J Cancer 2014;110:2524-30. 